\title{
Use of Olive Mill Wastewater as Substrate for Laccase Production by Fungi ${ }^{\dagger}$
}

\author{
Zeyneb BOUCHERIT ${ }^{1,}{ }^{*}$, Raouf ZEHIOUA ${ }^{2}$ and Aicha MECHAKRA ${ }^{3}$ \\ 1. Department of Microbiology, University of Constantine 1, Constantine 25017, Algeria \\ 2. Laboratoire de l'Ingénierie des Procédés de l'Environnement, Université Constantine 3, Constantine \\ 25017, Algeria \\ 3. Faculty of Nature and Life Sciences, Department of Microbiology, Laboratory of Biology and \\ Environment, University of Mentouri Brothers-Constantine 1, Constantine 25017, Algeria \\ * Correspondence: zeyneb.boucherit@umc.edu.dz \\ + Presented at 1st International Electronic Conference on Applied Sciences, 10-30 November 2020; \\ Available online: https://asec2020.sciforum.net/.
}

Published: 10 November 2020

\begin{abstract}
The annual Olive oil production in Mediterranean countries is estimated to over $95 \%$ of the world cultivation. Nevertheless this process generates large amounts of wastes including Olive Mill Wastewaters (OMW) with approximately $3 \times 10^{7} \mathrm{~m}^{3}$ per year which pose a critical problem for environment due to their colossal concentration of salinity, organic matter and phenolic compounds responsible of the phytotoxicity and microbial growth inhibitory effects. For these reasons, most attention has been agreed to determinate the best strategy to reduce phenol content of OMW. Laccases were recognized as main enzymes implicated in the degradation of phenolics in OOW alongside of this the use of halotolerant fungi offer potential applications for production of enzymes able to retain good activity in saline environments. The aim of this work was to investigate the ability of halophilic fungi isolated from saline soil to produce laccase and degrade OMW on agar plate method. For this purpose, 23 strains of fungi are isolated from the sebkha's soil of Ain'Ezzmoul, Algeria on PDA plates. Same medium supplemented with Guaiacol was used to investigate the laccase production. Then the three positif laccasic strains obtained (GS7, GS15 and VS1) were examined for their ability to grow and decolorize OMW. Therefore, a three phasic OMW were collected and prepared with several concentrations and solidified with $2 \%$ Agar. The strains GS7, GS15 and VS1 respectively were able to grow at OWW up to $50 \%, 40 \%$ and $100 \%$ but only GS15 showed a complete decolourisation halo. This latter was tested to laccase production on OMW liquid medium and shows enzymes activities $61.05 \mathrm{U}$ and $67.72 \mathrm{U}$ on respectively $10 \%$ and $20 \%$ of unsupplemented OMW.
\end{abstract}

Keywords: Olive oil mill ; phenol ; laccase ; fungi

Publisher's Note: MDPI stays neutral with regard to jurisdictional claims in published maps and institutional affiliations.

(C) 2020 by the authors. Submitted for possible open access publication under the terms and conditions of the Creative Commons Attribution (CC BY) license (http://creativecommons.org/licenses/by/4.0/). 